\title{
Sodium tanshinone IIA sulfonate protects rat myocardium against ischemia-reperfusion injury via activation of PI3K/Akt/F0X03A/Bim pathway
}

\author{
Mei-qi ZHANG ${ }^{1, \#}$, Yue-liang ZHENG ${ }^{1, \#}$, Huan $\mathrm{CHEN}^{1}$, Jian-feng $\mathrm{TU}^{1}$, Ye $\mathrm{SHEN}^{1}$, Jun-ping GUO ${ }^{1}$, Xiang-hong YANG ${ }^{1}$, Shu-ren \\ $\mathrm{YUAN}^{2}$, Liang-zhong $\mathrm{CHEN}^{2}$, Jing-jie $\mathrm{CHAl}^{2}$, Jian-hong $\mathrm{LU}^{2}$, Chang-lin $\mathrm{ZHAI}^{2}$ * \\ ${ }^{1}$ Department of Emergency Intensive Care Unit, Zhejiang Provincial People's Hospital, Hangzhou 310014, China; ${ }^{2}$ Department of Car- \\ diology, First People's Hospital of Tongxiang, Hangzhou 314500, China
}

Aim: To investigate the mechanisms underlying the protective effects of sodium tanshinone IIA sulfonate (STS) in an ischemiareperfusion (I/R)-induced rat myocardial injury model.

Methods: Male SD rats were iv injected with STS, STS+LY294002, or saline (NS) for $15 \mathrm{~d}$. Then the hearts were subjected to 30 min of global ischemia followed by $2 \mathrm{~h}$ of reperfusion. Cardiac function, infarction size and area at risk were assessed. Cell apoptosis was evaluated with TUNEL staining, DNA laddering and measuring caspase-3 activity. In addition, isolated cardiomyocytes of neonatal rats were pretreated with the above drugs, then exposed to $\mathrm{H}_{2} \mathrm{O}_{2}(200 \mu \mathrm{mol} / \mathrm{L})$ for $1 \mathrm{~h}$. Cell apoptosis was detected using flow cytometric assay. The levels of $\mathrm{p}-\mathrm{Akt}, \mathrm{p}-\mathrm{FOXO} \mathrm{AA}$ and Bim were examined with immunoblotting.

Results: Compared to NS group, administration of STS $(20 \mathrm{mg} / \mathrm{kg})$ significantly reduced myocardial infarct size $(40.28 \% \pm 5.36 \%$ in STS group vs 59.52\% $\pm 7.28 \%$ in NS group), and improved the myocardial function as demonstrated by the increased values of $\mathrm{d} p / \mathrm{d} t_{\text {max }}$, LVDP and coronary flow at different reperfusion time stages. Furthermore, STS significantly decreased the rate of apoptotic cells (15.11\% $\pm 3.71 \%$ in STS group vs 38.21\% $\pm 7.83 \%$ in NS group), and reduced caspase-3 activity to nearly a quarter of that in NS group. Moreover, STS significantly increased the phosphorylation of Akt and its downstream target FOXO3A, and decreased the expression of pro-apoptotic gene Bim. Co-treatment with the PI3K inhibitor LY294002 (40 mg/kg) partially countered the protective effects induced by STS treatment. In isolated cardiomyocytes, STS exerted similar protective effects as shown in the ex vivo I/R model.

Conclusion: STS pretreatment reduces infarct size and improves cardiac function in an I/R-induced rat myocardial injury model via activation of Akt/FOXO3A/Bim-mediated signal pathway.

Keywords: sodium tanshinone IIA sulfonate; heart attack; myocardial ischemia-reperfusion; apoptosis; Akt; FOXO3a; Bim; PI3K; LY294002

Acta Pharmacologica Sinica (2013) 34: 1386-1396; doi: 10.1038/aps.2013.91; published online 30 Sep 2013

\section{Introduction}

Myocardial ischemia-reperfusion (I/R) injury is a major complication that occurs during heart attack, cardiopulmonary bypass surgery, and heart transplantation ${ }^{[1]}$, which ultimately leads to an irreversible fatal injury. Apoptosis plays a crucial role in cardiac dysfunction following acute myocardial infarction $^{[2]}$. Various strategies aimed at preventing or mitigating the extent of apoptosis have been attempted to protect the heart against I/R injury. However, all attempts to date have exhibited limited efficacy. Developing new therapies for

\footnotetext{
\#The two authors contributed equally to this work.

* To whom correspondence should be addressed.

E-mail changlinzhai_2012@yahoo.com.cn

Received 2012-12-09 Accepted 2013-06-24
}

myocardial I/R injury represents an urgent and significant research interest ${ }^{[3]}$.

Apoptotic cell death can be induced by ischemia alone due to a lack of oxygen supply, and reperfusion accelerates the apoptotic death process that is initiated during the preceding ischemic period ${ }^{[4]}$. Thus, studies have suggested that the apoptotic component of cell death is either triggered or accelerated during the reperfusion phase ${ }^{[5]}$. I/ $R$ has been shown to activate the pro-survival PI3K/Akt kinase signaling cascades, which have been implicated in cellular survival, through their recruitment of protective anti-apoptotic proteins. The serine/ threonine-specific protein kinase Akt [also known as protein kinase B) is a potent survival factor and is one of the primary kinases that is activated by PI3K, which, in turn, is activated by several myocardial protective ligand-receptor systems, 
including insulin and angiotensin II. Important downstream targets of Akt are members of the FOXO subfamily of forkhead transcription factors. This FOXO subfamily includes at least the following three members: FOXO1, FOXO3A, and FOXO4, all of which can be inactivated by Akt-dependent phosphorylation ${ }^{[6]}$. Among these three FOXO members, FOXO3A regulates cellular apoptosis by inducing the proapoptotic gene Bim in cancer cells, neurons, and endothelial progenitor cells ${ }^{[7,8]}$. Weston et al ${ }^{[9]}$ demonstrated that the inhibition of the PI3K/Akt pathway resulted in an increase in Bim expression, implying that by phosphorylating Akt, the activation of the PI3K-Akt pathway exerts an inhibitory influence on Bim expression, thereby preventing apoptosis.

Sodium tanshinone IIA sulfonate (STS $)^{[10]}$ is a water-soluble derivative of tanshinone $\operatorname{IIA}^{[11,12]}$, which is a major lipophilic component that is extracted from the root of the Salvia miltiorrhiza (Fam Labiatae) plant. This plant is well-known in traditional Chinese medicine and is used for the treatment of various cardiovascular diseases, such as myocardial infarctions and coronary heart disease. Recent studies ${ }^{[13,14]}$ have revealed that STS elicits protective effects on cardiomyocytes against oxidative stress-mediated apoptosis in preclinical experiments. Hence, the cardioprotective effect of STS may represent therapeutic potential for clinical applications.

Given the cardioprotective effect of the PI3K/Akt pathway in the I/R rat model, we hypothesized that the cardioprotective effect of STS might involve the activation of the PI3K/Akt pathway. Therefore, the aims of this study were to: investigate the cardioprotective effect of STS against myocardial I/R injury in the rat heart and: to identify whether the underlying protective mechanisms are associated with the Akt/FOXO3A/ Bim-mediated apoptosis pathway both in vivo and in vitro.

\section{Materials and methods}

\section{Animal maintenance and treatment}

All procedures and studies in this article were in compliance with the "Guide for the Care and Use of Laboratory Animals" prepared by the National Academy of Science and published by the US National Institutes of Health (Publication No 85-23, revised 1996). The experimental protocol was approved by the Committee on Animal Experiments of Zhejiang University School of Medicine. Male Sprague-Dawley rats weighing between 250 and $300 \mathrm{~g}$ were obtained from the Experimental Animal Center of Hangzhou, Zhejiang Province (China) and were allowed free access to laboratory chow and tap water in day-night quarters at $25^{\circ} \mathrm{C}$.

The rats were randomized into the following three groups, which included eight animals each: (1) I/R rats pretreated with saline (NS group); (2) I/R rats pretreated with STS at a dose of $20 \mathrm{mg} / \mathrm{kg}$ (STS group); and (3) I/R rats co-pretreated with $20 \mathrm{mg} / \mathrm{kg}$ STS and $40 \mathrm{mg} / \mathrm{kg}$ PI3K inhibitor LY294002 (STS+LY294002 group).

\section{STS treatment}

STS was obtained from the Department of Pharmacology,
Zhejiang University of Traditional Chinese Medicine. It was dissolved in PBS at a concentration of $10 \mathrm{mg} / \mathrm{mL}$ and was prepared freshly every day. Each animal received intravenous injection of STS once per day at the same time. After $15 \mathrm{~d}$ of injections, the ex vivo ischemic induction and isolation of the hearts were performed.

\section{PI3K activity inhibition}

To investigate the role of the PI3K/Akt pathway in myocardial I/R-induced apoptosis, a specific PI3K inhibitor was injected. The inhibitor, LY294002, was purchased from BIOMOL Research Laboratories (Plymouth Meeting, PA) and dissolved in DMSO and PBS. LY294002 (40 mg/ kg dissolved in 25\% dimethylsulfoxide and diluted 1:10 in PBS) was administered intravenously in the tail vein at $30 \mathrm{~min}$ prior to the ischemia and reperfusion processes.

\section{Isolated working heart preparation}

The isolated myocardial I/ R model was established as previously described ${ }^{[15]}$. Briefly, the rats were anesthetized using pentobarbital sodium ( $80 \mathrm{mg} / \mathrm{mL} \mathrm{ip})$ and a heparinized $0.9 \%$ saline solution (500 IU/kg per day, iv). After a sufficient depth of anesthesia, thoractomy was performed, and the hearts were perfused in the retrograde Langendorff mode at $37^{\circ} \mathrm{C}$ at a constant perfusion pressure of $100 \mathrm{cmH}_{2} \mathrm{O}(10 \mathrm{kPa})$ for 5 min washout period. A modified Krebs-Henseleit bicarbonate buffer (KHB), containing (in mmol/L) $118 \mathrm{NaCl}, 4.7$ $\mathrm{KCl}, 1.7 \mathrm{CaCl}_{2}, 25 \mathrm{NaHCO}_{3}, 1.2 \mathrm{KH}_{2} \mathrm{PO}_{4}, 1.2 \mathrm{MgSO}_{4}$, and 10 glucose, was used in this perfusion buffer. The Langendorff preparation was switched to the working mode following the washout period, as previously described ${ }^{[16,17]}$. The working mode was performed by switching the flow from the aortic root to the left atrium with a constant preload of $17 \mathrm{cmH}_{2} \mathrm{O}$ and an afterload of $100 \mathrm{cmH}_{2} \mathrm{O}^{[16,17]}$. After reaching a steadystate of cardiac function, the baseline functional parameters were recorded. The circuit was then switched back to the retrograde mode, and the hearts were perfused for $15 \mathrm{~min}$ with KHB buffer. Next, the hearts were subjected to global ischemia for $30 \mathrm{~min}$ followed by $2 \mathrm{~h}$ of reperfusion. The first $10 \mathrm{~min}$ of reperfusion were performed in the retrograde mode to allow for postischemic stabilization. Subsequently, the reperfusion was performed in the anterograde-working mode to allow for the assessment of functional parameters, which were recorded at 30, 60, 90, and $120 \mathrm{~min}$ of reperfusion.

\section{Determination of cardiac function}

Aortic pressure was measured using a pressure transducer (Micro-Med, USA) connected to a sidearm of the aortic cannula, and the signal was amplified using a Heart Performance Analyzer (model 400; Micro-Med, USA). The heart rate (HR), the first derivative of developed pressure $(\mathrm{d} p / \mathrm{d} t)$ and the left ventricular developed pressure (LVDP; systolic pressure minus the end-diastolic pressure in $\mathrm{mmHg}$ ) were all derived or calculated from the continuously obtained pressure signal. Coronary flow (CF) was measured by a timed collection of the coronary effluent dripping from the heart. 


\section{Estimation of infarct size and area at risk}

At the end of reperfusion, a $1 \%(w / v)$ solution of triphenyl tetrazolium chloride (TTC) in phosphate buffer was infused into the aortic cannula at $37^{\circ} \mathrm{C}$. The hearts were quickly excised and stored at $-70^{\circ} \mathrm{C}$. Sections of frozen heart were fixed in $10 \%$ formalin, placed between two cover slips, and digitally imaged using a Epson scanner. The infarcted area remained unstained, whereas the non-infarcted area stained red. Negatively stained areas indicated infarcted myocardium. Next, the coronary arteries were retied at the site of previous occlusion, and the hearts was perfused with a $2 \%$ solution of Evans blue dye to delineate the ischemic area (area at risk). The areas of infarcted, ischemic and non-ischemic myocardium were measured and calculated using NIH image analysis software, and from these measurements, the infarcted size (IS) was calculated as a percentage of the area at risk (AAR). The AAR was expressed as a percentage of the left ventricles (LVS).

\section{Histological examination}

The frozen left ventricle fixed by formalin were then embedded in paraffin and cut into transverse sections at a thickness of $6 \mu \mathrm{m}$, with a routine follow-up procedure. The sections were stained with hematoxylin and eosin for light microscopic examination. An observer that was blinded to the experimental conditions of animals recorded the data.

Determination of cardiomyocyte apoptosis and caspase-3 activity The terminal deoxynucleotidyl transferase-mediated dUTPbiotin nick end-labeling (TUNEL) assay was performed, according to the manufacturer's protocols (Roche, Switzerland). The cells exhibiting clear nuclear labeling were defined as TUNEL positive cells. For each slide, the color images of 10 separate fields were captured randomly and digitized. The numbers of TUNEL positive cells in each group were counted separately using ImageJ software.

The caspase-3 activity was measured using ApoAlert Caspase-3 Assay Plate (Clontech, Mountain View, CA, USA), according to the manufacturer's protocols. Substrate cleavage was monitored fluorometrically using a SpectraMax Gemini spectrophotometer (Molecular Devices, Sunnyvale, CA, USA) at excitation and emission wavelengths of 350 and $450 \mathrm{~nm}$, respectively.

\section{Subcellular cytoplasmic and mitochondrial fractionation}

For subcellular fractionation, cell lysates were produced using a glass tissue grinder (Wheaton, Millville, NJ, USA). The lysates were centrifuged at $750 \times g$ for $10 \mathrm{~min}$ at $4^{\circ} \mathrm{C}$ and subsequently at $8000 \times g$ for $20 \mathrm{~min}$ at $4^{\circ} \mathrm{C}$. The resulting pellets were used to obtain the mitochondrial fraction. The supernatant was centrifuged further at $100000 \times g$ for $60 \mathrm{~min}$ at $4^{\circ} \mathrm{C}$ and was used for the analysis of the cytosolic fraction.

\section{Quantitative real-time PCR for detection of Bim mRNA}

Total RNA was isolated from the left ventricles by TRIZOL (Gibco-BRL, USA), according to the manufacturer's protocols, and its quality was evaluated on a $1 \%$ denaturing aga- rose gel to ensure the presence of the $28 \mathrm{~S}$ and $18 \mathrm{~S}$ ribosomal bands. The total RNA concentrations were determined by spectrophotometry. A total of $1 \mu \mathrm{g}$ of RNA was incubated in a reverse transcription mixture at $42^{\circ} \mathrm{C}$ for $50 \mathrm{~min}$, followed by PCR amplification using the specific primers (synthesized by Invitrogen Company, USA). The following primers were used: Bim FW: 5'-CCCCTACCTCCCTACAGACA-3', RV: 5'-GACGGAAGATGAATCGTAACAG-3'; GAPDH FW: 5'-ATCCGTTGTGGATCTGACATG-3', RV: 5'-TGGTCCAGGGTTTCTTACTCC-3'. The PCR conditions were $95^{\circ} \mathrm{C}$ for $15 \mathrm{~s}$ followed by 30 cycles of amplification consisting of $95^{\circ} \mathrm{C}$ for $5 \mathrm{~s}$ and $60^{\circ} \mathrm{C}$ for $30 \mathrm{~s}$. The reactions were performed in triplicate, and the results were averaged. Each value was normalized to GAPDH mRNA levels to control for variations in the amount of input cDNA. The relative expression levels of the genes compared with the control group were calculated using the $2^{\Delta \Delta \mathrm{Ct}}$ method.

\section{Extraction of protein and Western blot assay}

Freshly frozen myocardial tissue samples were homogenized in RIPA lysis buffer, and centrifuged to extract the total protein. The Bradford assay (Bio-Rad Laboratories, USA) was used to quantify protein concentrations. Total protein (50 $\mu \mathrm{g})$ was separated on $12 \%$ SDS-PAGE gels and transferred to nitrocellulose filter membranes. Primary antibodies against Akt, phosphorylated Akt (p-Akt), FOXO1, p-FOXO1, FOXO3A, p-FOXO3A, FOXO4, p-FOXO4, cytochrome $c$, and Bim (Abcam, UK) were used at an appropriate dilution ratio, followed by peroxidase-coupled secondary antibodies (Sigma, USA, 1:10000). The blots were visualized by enhanced chemiluminescence system using an ECL kit (Roche, USA). The signals were quantified by densitometry using imaging software (BioSens Digital Imaging 5, Shanghai Bio-Tech Inc, Shanghai, China).

\section{Neonatal rat cardiac cell cultures}

Cardiac cells were isolated from 1-to-3-d-old neonatal Wistar rats and were cultured as previously described ${ }^{[18]}$. Briefly, the rats were sacrificed and their hearts were excised. After scalpel homogenization, the heart tissue samples were incubated in $0.25 \%(w / v)$ trypsin overnight at $4^{\circ} \mathrm{C}$ after treatment with $0.1 \%(w / v)$ collagenase for $20 \mathrm{~min}$ at $37^{\circ} \mathrm{C}$. Cardiomyocytes were enriched by centrifugation and plated in Dulbecco's modified Eagle's medium (DMEM) supplemented with 10\% $(v / v)$ fetal calf serum at $37^{\circ} \mathrm{C}$ and $5 \%(v / v) \mathrm{CO}_{2}$ for $72 \mathrm{~h}$. The cardiomyocytes were allowed to adhere overnight in serumdeprived medium prior to treatment.

\section{Drug treatment and cardiomyocyte injury induced by exposure to $\mathrm{H}_{2} \mathrm{O}_{2}$}

To evaluate the protective effects of STS, cells were pretreated for $12 \mathrm{~h}$ with $3 \mu \mathrm{g} / \mathrm{mL}$ STS alone or $3 \mu \mathrm{g} / \mathrm{mL}$ STS and 10 $\mu \mathrm{mol} / \mathrm{L}$ LY294002 at $1 \times 10^{6}$ cells/well in six-well plates, and washed twice with D-Hank's solution prior to the addition of $\mathrm{H}_{2} \mathrm{O}_{2}$. The cardiomyocytes were then exposed to $200 \mu \mathrm{mol} / \mathrm{L}$ $\mathrm{H}_{2} \mathrm{O}_{2}$ for $1 \mathrm{~h}^{[19]}$. The control cells were also incubated under 
identical conditions.

\section{Flow cytometric analyses for apoptosis}

Apoptotic cells were detected by annexin V and 7-AAD double-labeling following the manufacturer's protocol, as previously described ${ }^{[18]}$. Briefly, cells were harvested at the indicated time periods, washed twice with cold phosphatebuffered saline (PBS), and then re-suspended in $500 \mu \mathrm{L}$ binding buffer. Next, the cells were stained with $5 \mu \mathrm{L}$ of annexin V-fluorescein isothiocyanate (FITC) and $5 \mu \mathrm{L}$ of 7-AAD for 15 min at room temperature in the dark. Flow cytometry (BD FACSAria II) was used to assess the percentage of cell apoptosis.

\section{Statistical analysis}

The results were analyzed using the SPSS statistical package (ver 12.0; IBM, USA) and are presented as the mean values \pm SEM. The data were compared using Tukey's honest significance test and a one-way analysis of variance (ANOVA). $P$-values $<0.05$ were considered statistically significant.

\section{Results}

\section{Protective effects of STS on myocardial function}

To evaluate the protective effects of STS on heart against ischemic injury, we established a rat model of in vitro global I/R. As shown in Figure 1A, pre-treatment with STS significantly reduced the myocardial infarction sizes after I/ R compared with that observed in the NS group $(40.28 \% \pm 5.36 \%$ vs $59.52 \% \pm 7.28 \%, P<0.05)$. This effect was partially abrogated by co-treatment with LY294002 (50.76\% $\pm 5.49 \%$ in STS+LY294002 group vs $40.28 \% \pm 5.36 \%$ in STS group, $P<0.05)$. However, AAR, which was defined as the myocardial tissue within the perfusion bed distally to the culprit lesion of the infarct-related coronary artery, was similar among all of the groups.

Myocardial functions $\left(\mathrm{d} p / \mathrm{d} t_{\max }, \mathrm{LVDP}\right.$, and $\mathrm{CF}$ ) were significantly enhanced both at the baseline (prior to reperfusion) as well as during reperfusion in the STS group compared with the NS group. However, no significant difference was observed in HR (Figure 1B) among the three groups during I/R. The STS group exhibited a significant increase in $\mathrm{d} p / \mathrm{d} t_{\max }$ both at baseline and after $30 \mathrm{~min}$ of ischemia (Figure 1C) throughout the reperfusion period compared with the control (2984 \pm 274 vs $2493 \pm 209 \mathrm{mmHg} / \mathrm{s} 30$ min post reperfusion). A similar pattern was observed in LVDP as well as in coronary flow (Figure 1D and 1E) in the STS group compared with the control. As expected, co-treatment with LY294002 in STS+LY294002 group in part abolished the protective effect elicited by STS treatment (Figure 1C-1E). These results indicated the PI3K/Akt-dependent cardioprotective effects of STS.

\section{I/R-induced apoptosis is alleviated by STS pretreatment}

The representative photograph show that TUNEL-positive cardiomyocytes were more frequently observed in the NS group and STS+LY294002 group compared to the STS group. Semiquantitative analysis revealed that the incidence of TUNEL- positive cells was significantly reduced in the STS group compared to the NS group $(15.11 \% \pm 3.71 \%$ vs $38.21 \% \pm 7.83 \%$, $P<0.05)$. When co-treated with LY294002 in the STS+LY294002 group, the percentage of TUNEL-positive cells was significantly increased compared to the STS group $(22.08 \% \pm 5.22 \%$ vs $15.11 \% \pm 3.71 \%, P<0.05$ ) (Figure $2 \mathrm{~A}$ ).

Mitochondrial dysfunction plays an important role in ischemia-reperfusion-induced cardiomyocyte apoptosis injury. Thus, we further assessed cytosolic and mitochondrial cytochrome $c$ protein levels by immunoblotting analysis. As indicated in Figure 2B, we observed that in the NS group, cytochrome $c$ was predominantly found within the cytosolic fraction, reflecting an active apoptotic process. Compared to the NS group, a significant decrease in cytochrome $c$ within the cytosolic fraction accompanied by an increase within the mitochondrial fraction was observed in the STS group. Moreover, co-treatment with LY294002 reversed the effect of STS on the subcellular distribution of cytochrome $c$ between the cytosolic and mitochondrial fractions. However, the reversal did not reach the level observed in the NS group. There was no significant difference in the total expression levels of cytochrome $c$ between the three groups (Figure 2B).

The internucleosomal DNA fragmentation of cardiomyocytes represents a hallmark of apoptotic cell death. Negligible levels of DNA laddering were observed in the STS group, whereas DNA laddering was clearly detected in the NS and STS+LY294002 groups after $2 \mathrm{~h}$ of reperfusion. STS treatment significantly decreased the appearance of DNA laddering induced by I/R, which was counteracted by co-treatment with LY294002 (Figure 2C).

As an executioner caspase, caspase- 3 is activated in apoptotic cells both by extrinsic (death ligand) and intrinsic (mitochondrial) pathways. The caspase-3 zymogen exhibits virtually no activity until it is cleaved by an initiator caspase following apoptotic signaling events. As shown in Figure $2 \mathrm{D}$, the caspase-3 activity was significantly reduced in the STS group compared to the NS group, and co-treatment with LY294002 in the STS+LY294002 group significantly enhanced caspase-3 activity compared to that observed in STS group. These results demonstrated that the administration of STS significantly alleviated the I/R-induced myocardial cell apoptotic injury in the rats via the activation of a PI3K-dependent signaling pathway.

The PI3K/Akt pathway is involved in the protective effect of STS STS treatment significantly increased the phosphorylated Akt (p-Akt) levels in myocardial tissues that were exposed to I/R compared to the NS group. The levels of p-Akt were significantly reduced when the PI3K/Akt inhibitor LY294002 was applied. The total Akt levels ( $\mathrm{t}-\mathrm{Akt}$ ) remained unchanged among the three groups (Figure 3 ). These results imply that the PI3K/Akt pathway is involved in the STS-induced protective effects during I/R. STS treatment triggered PI3K activity that further activated the downstream potent survival factor phosphorylated Akt. 

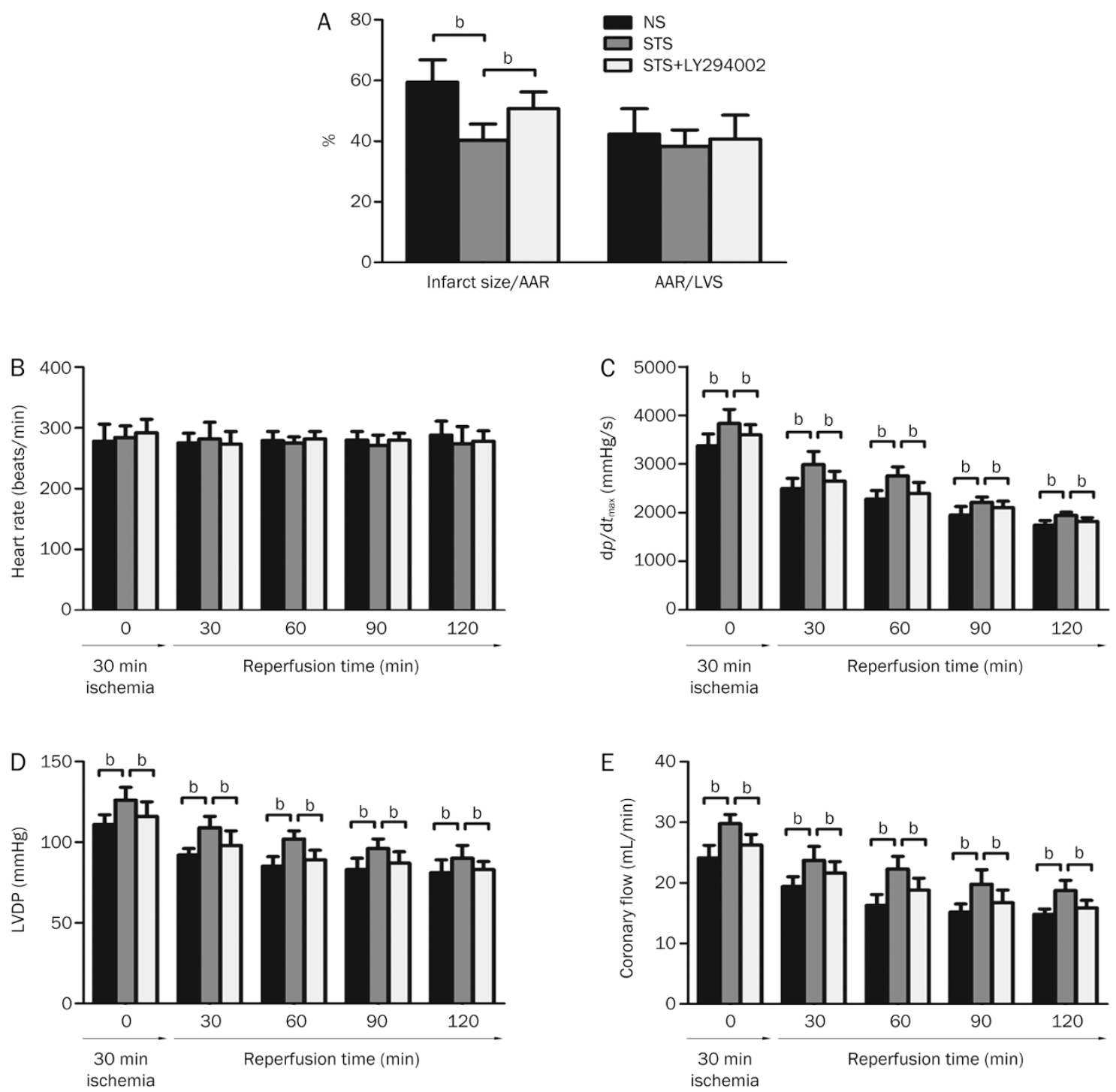

Figure 1. The effects of sodium tanshinone IIA sulfonate (STS) on infarct size, area at risk (AAR) (A), heart rate (HR) (B), the first derivative of developed pressure ( $\left.\mathrm{dp} / \mathrm{d} t_{\max }\right)(\mathrm{C})$, left ventricular developed pressure (LVDP) (D) and coronary flow (CF) (E). The isolated hearts from NS-, STS-, and STS+LY294002-treated rats were subjected to $30 \mathrm{~min}$ of global ischemia followed by $2 \mathrm{~h}$ of reperfusion. The results are expressed as the mean \pm SEM. ${ }^{\mathrm{b}} \mathrm{P}<0.05$ was considered statistically significant among each group. $n=8$ animals in each group.

STS treatment induces F0X03A phosphorylation but elicits no effect on FOXO1 and FOXO4

Mammalian forkhead members of the class O (FOXO) transcription factors, including FOXO1, FOXO3a, and FOXO4, are implicated in the regulation of several biological processes, such as stress resistance, metabolism, cell cycle, and apoptosis. To investigate which of the FOXO family member might be activated by STS treatment, we assessed the phosphorylation levels of each FOXO family member, including FOXO1, FOXO3A, and FOXO4. As shown in Figure 4, the levels of phosphorylated FOXO3A were significantly elevated in the STS group compared with that observed in the NS group (7.24 \pm 1.42 -fold, $P<0.05)$. Co-treatment with LY294002 in the STS+LY294002 group significantly reduced the expression of phosphorylated FOXO3a in STS group, although the levels were still increased compared to that observed in NS group (2.94 \pm 0.63 -fold, $P<0.05)$. Notably, the administration of STS did not alter the levels of phosphorylated FOXO1 and FOXO4 proteins among each group. Pre-treatment with STS alone or with LY294002 did not affect the total levels of FOXO1, FOXO3A or FOXO4 proteins. These results indicated that the protective effect of STS was associated with Akt-mediated FOXO3a signaling pathway, but not FOXO1 or FOXO4.

STS down-regulates Bim expression in a PI3K-dependent signaling pathway

The down-regulation of both mRNA and protein levels of Bim were observed in the STS group compared to the NS group. Moreover, as expected, co-treatment with LY294002 increased the mRNA and protein expression levels of Bim, which was 

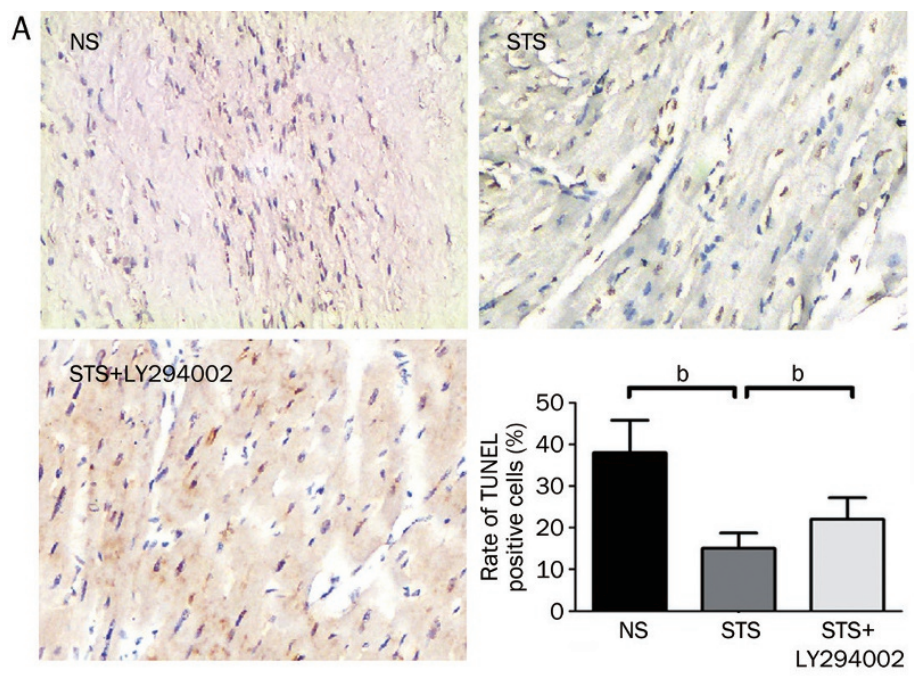

C

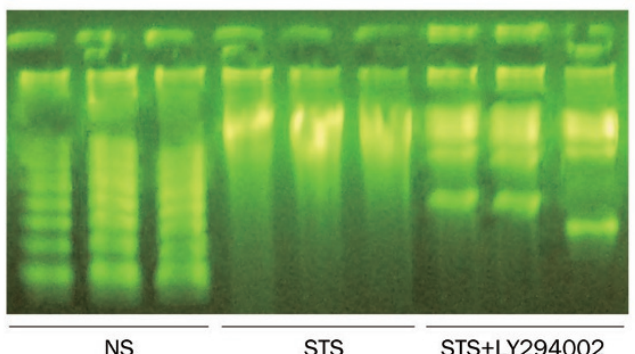

B
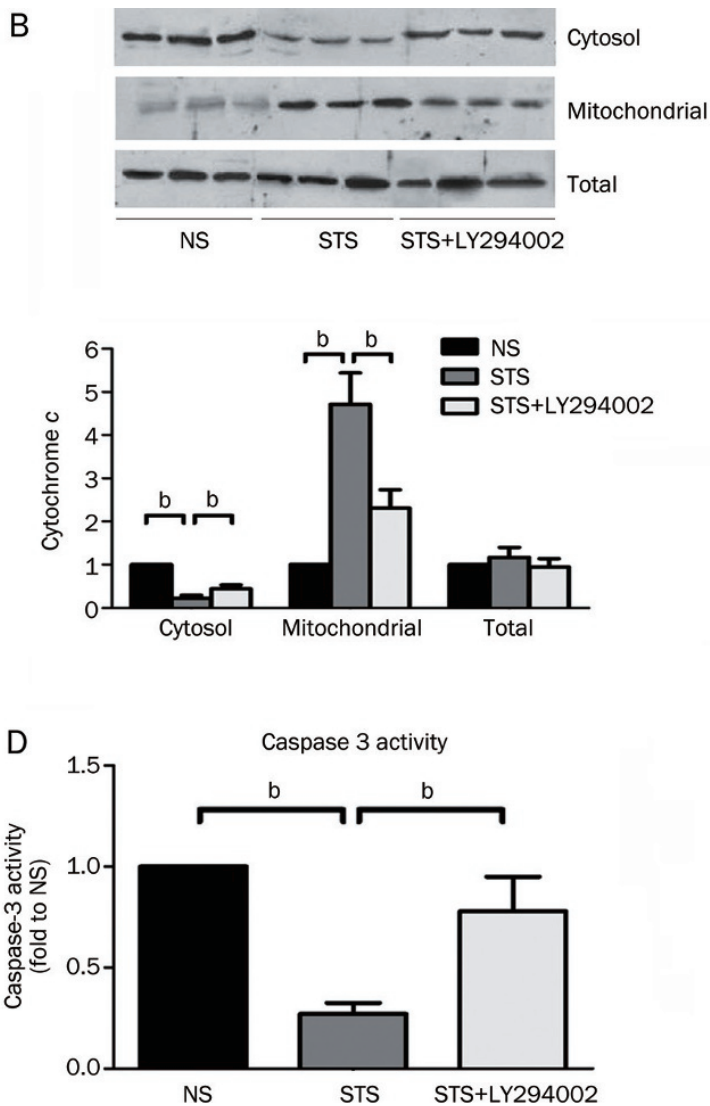

Figure 2. The effect of STS on myocardial apoptosis after ischemia/reperfusion (I/R) injury. (A) Representative photomicrographs demonstrating TUNEL staining for apoptotic cells in the rat heart at $2 \mathrm{~h}$ after reperfusion in the NS, STS, and STS+LY294002 groups. The effects on the severity of cardiac apoptosis are shown in an average quantitative analysis of the rate of TUNEL-positive cells. The data are represented as the mean $\pm S E M$. $n=8$ for each group. ${ }^{b} P<0.05$ was considered statistically significant among each group. (B) Cytochrome $c$ levels in the cytosol, mitochondrial and total fraction were analyzed by WB and semi-quantitatively estimated as the fold-change relative to the NS group. (C) The detection of internucleosomal DNA fragmentation using gel electrophoresis and Biotekepeter Ultra power staining in the NS, STS, and STS+LY294002 groups. (D) The effects of STS with or without LY294002 treatment on caspase 3 activity in area at risk zone of the cardiac tissues at $2 \mathrm{~h}$ after reperfusion, quantitatively estimated as the fold-change relative to the NS group. ${ }^{\mathrm{b}} \mathrm{P}<0.05$ is considered significant difference among each groups.
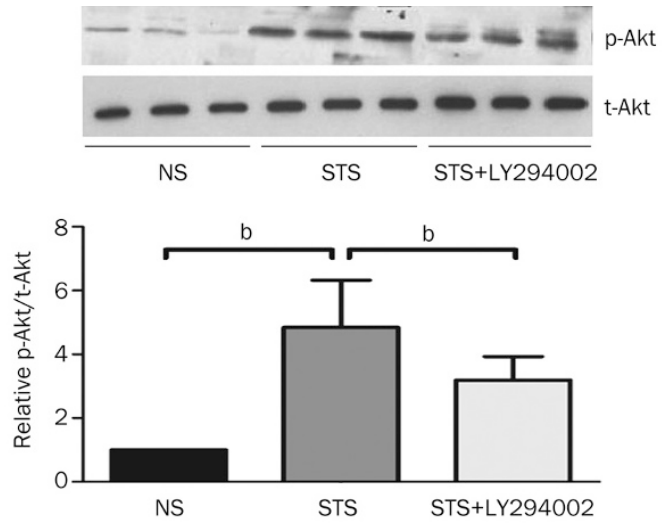

Figure 3. The effect of STS on phosphorylated Akt levels. Top: representative WB from left ventricle tissue isolated from each group showing the effect of STS on the levels of phosphorylated (p)-Akt and total (t)-Akt. Bottom: the quantitative estimation of p-Akt levels is shown as the relative $\mathrm{p}$-Akt and t-Akt ratios in STS and STS+LY294002 groups compared with the NS group. ${ }^{\mathrm{b}} P<0.05$ was considered statistically significant among each group. $n=8$ animals in each group. still reduced compared to the NS group (Figure 5A). The representative micrographs of rat myocardial IHC staining of Bim at $2 \mathrm{~h}$ after reperfusion are shown in Figure 5B. The average nuclear and cytoplasmic staining intensity of Bim was significantly reduced compared with that observed in the NS group. Co-treatment with LY294002 increased the average nuclear and cytoplasmic staining intensity of Bim, which was still reduced compared to the NS group. These results indicated that pre-treatment with STS down-regulated the expression of Bim in a PI3K/Akt-dependent signaling pathway.

The in vitro effect of STS on phospho-Akt, phospho-F0X03A, and Bim expression

There were no significant differences in phospho-Akt, phospho-FOXO3A or Bim expression between the normal neonatal rat cardiac cells and the $\mathrm{H}_{2} \mathrm{O}_{2}$-induced control group. Pre-treatment with STS significantly increased the levels of phospho-Akt and phospho-FOXO3A but elicited no effect on the expression levels of total Akt and FOXO3A (Figure 6A). Moreover, STS decreased the expression of Bim at the mRNA 

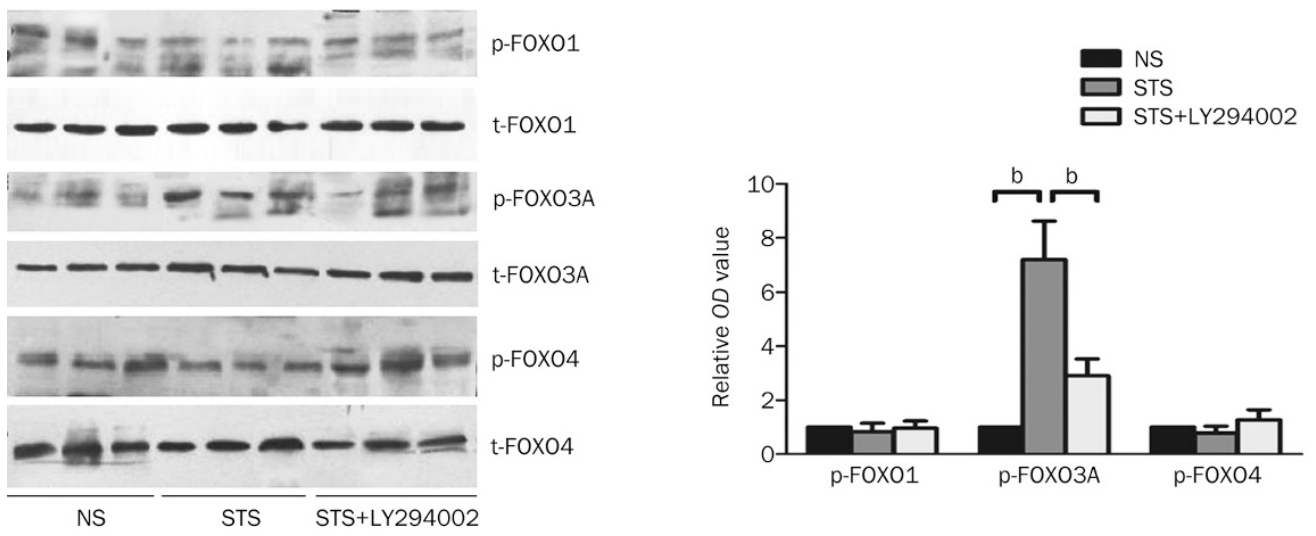

Figure 4. The effect of STS on phosphorylated FOXO1, FOXO3A, and FOXO4 levels. Left: a representative Western-blot from left ventricle tissues isolated from each group showing the effect of STS on the levels of phosphorylated (p)-FOXO1, p-FOXO3A, p-FOXO4, total ( $t$ )-FOXO1, t-FOXO3A, and t-FOXO4. Right: the quantitative estimation of p-FOXO1, p-FOXO3A, and p-FOXO4 levels are shown as the relative OD values in STS and STS+LY294002 groups compared to the NS group. ${ }^{b} P<0.05$ was considered statistically significant among each group. $n=8$ animals in each group.
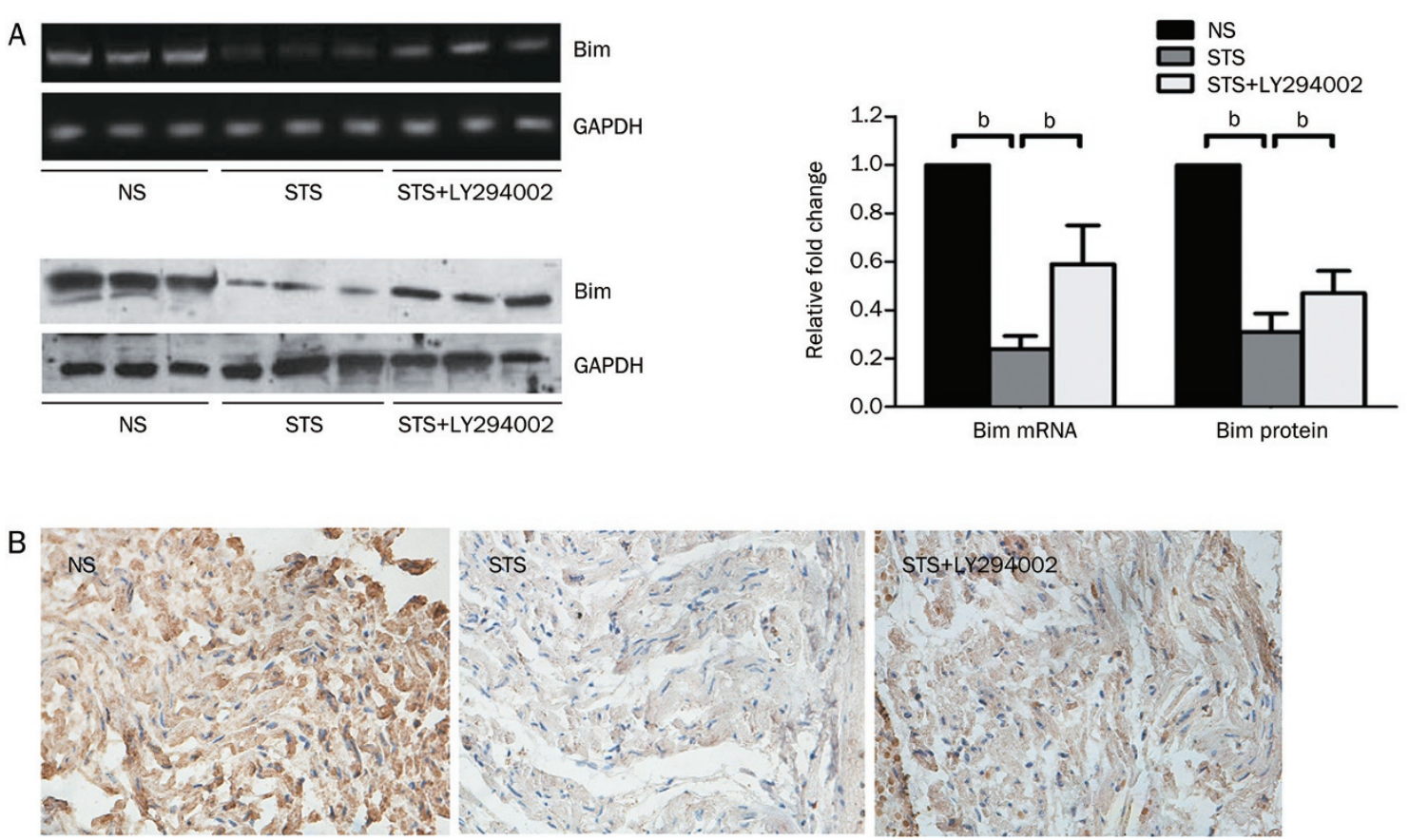

Figure 5. The effect of STS on Bim expression. (A) The expression levels of Bim mRNA (top) and protein (bottom) were analyzed using semiquantitative RT-PCR and Western blotting, respectively. The levels of GAPDH served as an internal control. The quantitative estimation of Bim mRNA and protein levels in the top right histogram are shown as the relative Bim mRNA and protein ratios in the STS and STS+LY294002 groups compared to the NS group. The expression levels of Bim mRNA and protein were measured in the myocardial tissue isolated from rats at $2 \mathrm{~h}$ after reperfusion. ${ }^{\mathrm{b}} P<0.05$ was considered as statistically significant among each group. $n=8$ animals in each group. (B) Representative photomicrographs show the immunohistochemical staining of Bim in the myocardial tissues isolated from rats at $2 \mathrm{~h}$ after reperfusion. STS attenuated Bim expression in the myocardial tissues (cytoplasm brown staining) at $2 \mathrm{~h}$ after reperfusion. Co-treatment with LY294002 resulted in elevated Bim staining compared to the STS group, although the staining was still weaker than that observed in the NS group.

and protein levels compared to $\mathrm{H}_{2} \mathrm{O}_{2}$-induced control group (Figure 6B). Co-treatment with STS and LY294002, however, partially counteracted the effects elicited by the addition of STS alone.
The in vitro effect of STS on apoptosis induced by $\mathrm{H}_{2} \mathrm{O}_{2}$

Flow cytometric analysis of Annexin V/7-AAD staining of neonatal rat cardiac cells revealed that $28.3 \%$ of cells were in the early stages of apoptosis at $1 \mathrm{~h}$ of exposure to $\mathrm{H}_{2} \mathrm{O}_{2}$. STS 
A
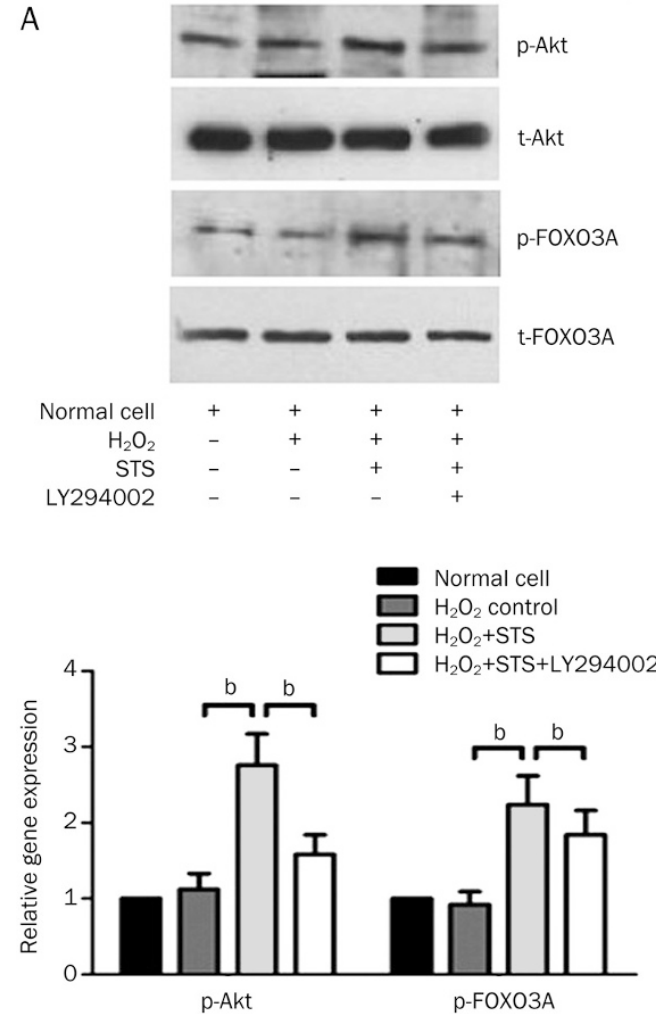

B

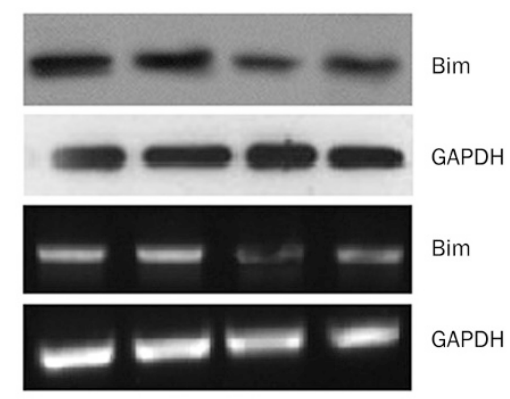

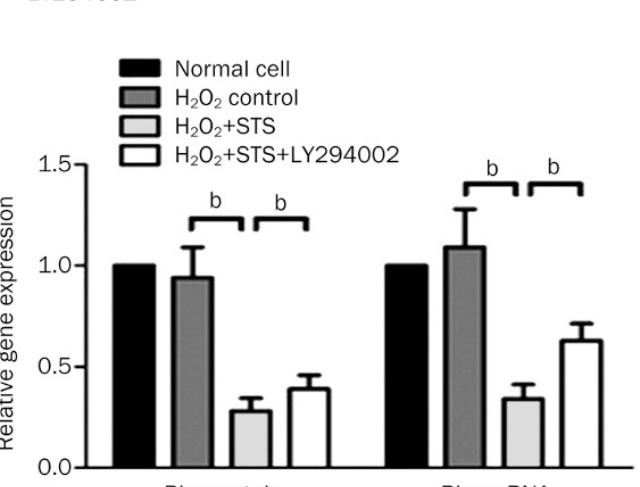

Bim protein

Figure 6. In vitro effect of STS on phospho-Akt, phospho-FOXO3A and Bim expression of neonatal rat cardiac cells induced by $\mathrm{H}_{2} \mathrm{O}_{2}$. (A) A representative Western blotting photograph showing the effect of STS on the levels of p-Akt, total-Akt, p-FOXO3A, and total-FOXO3A from each group. The quantitative estimation of phospho-Akt and phospho-FOXO3A levels was shown as the relative OD values in STS and STS+LY294002 groups compared with the normal and $\mathrm{H}_{2} \mathrm{O}_{2}$-induced control group. (B) The expression levels of Bim mRNA and protein were analyzed using a semi-quantitative RT-PCR and Western blotting, respectively. GAPDH served as an internal control. The quantitative estimation of Bim mRNA and protein levels in the histogram are shown as the relative Bim mRNA and protein ratios in the STS and STS $+L Y 294002$ groups compared to the normal and $\mathrm{H}_{2} \mathrm{O}_{2}$-induced control group.

treatment significantly decreased the extent of cardiomyocyte apoptosis compared to $\mathrm{H}_{2} \mathrm{O}_{2}$-induced control group $(15.92 \% \pm 3.36 \%$ vs $28.33 \% \pm 4.17 \%)$. However, apoptosis was significantly increased by co-treatment with STS and LY294002 compared to STS treatment alone $(21.59 \% \pm 3.87 \%$ vs $15.92 \% \pm 3.36 \%$ ) (Figure 7A, 7B).

The caspase-3 activity was significantly increased in cells exposed to $\mathrm{H}_{2} \mathrm{O}_{2}$ compared with the normal cell group. STS treatment significantly decreased caspase- 3 activity compared with the $\mathrm{H}_{2} \mathrm{O}_{2}$-induced control group. Co-treatment with STS and LY294002, however, significantly increased caspase-3 activity compared with STS treatment alone (Figure 7C).

\section{Discussion}

In this study, we demonstrated that in vivo and in vitro STS treatment elicited a protective effect against myocardial I/Rand $\mathrm{H}_{2} \mathrm{O}_{2}$-induced injury in rats and, furthermore, that the activation of a PI3K/Akt/FOXO3A/Bim-mediated antiapoptotic pathway is involved in this protective process. According to our findings, the protective effect of STS in cardiac function following reperfusion was reduced in the presence of LY294002 (Figure 1). Concurrently, STS significantly increased p-Akt levels in the myocardial tissue samples and the neonatal rat cardiac cells, an increase that was in part abolished by LY294002 treatment (Figure 3, 6). These results revealed that the PI3K/Akt signaling pathway is involved in the protective effect of STS. Moreover, Forkhead transcription factor FOXO3A, but not FOXO1 or FOXO4, was phosphorylated and might play a role as a downstream effector of Akt in mediating the protective effect of STS (Figure 4, 6). Apoptosis in cardiomyocytes induced by $\mathrm{I} / \mathrm{R}$ or exposure to $\mathrm{H}_{2} \mathrm{O}_{2}$ was significantly reduced when the cells were treated with STS but was enhanced following co-treatment with LY294002 (Figure $2,7)$. This protective mechanism of STS involves the inhibition of the apoptosis-related activation of caspase-3, cytochrome $c$ release, and the down-regulation of the Bim expression (Figure $2,5,6,7)$. Thus, our study indicated that the protective effect of STS is associated with the PI3K/Akt/FOXO3A/Bimmediated anti-apoptotic signaling pathway, a finding that is consistent with the results of previous studies ${ }^{[5,20,21,22]}$.

STS is a predominant effective derivative of Salvia miltiorrhiza (known as "Danshen" in Traditional Chinese Medicine). Experimental and clinical evidence have shown that STS reduces heart infarct size and protects cardiac tissue against I/R injury ${ }^{[23,24]}$. Salvianolic acid A (SAA), another major active component of Danshen, elicits a marked protective effect on 

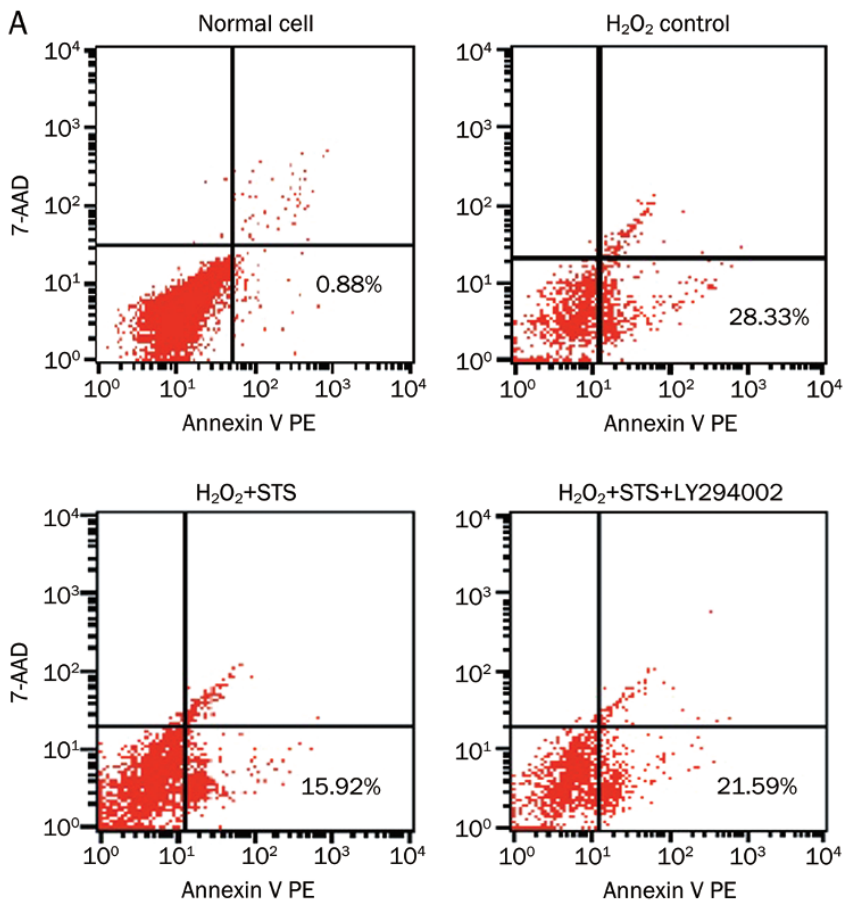
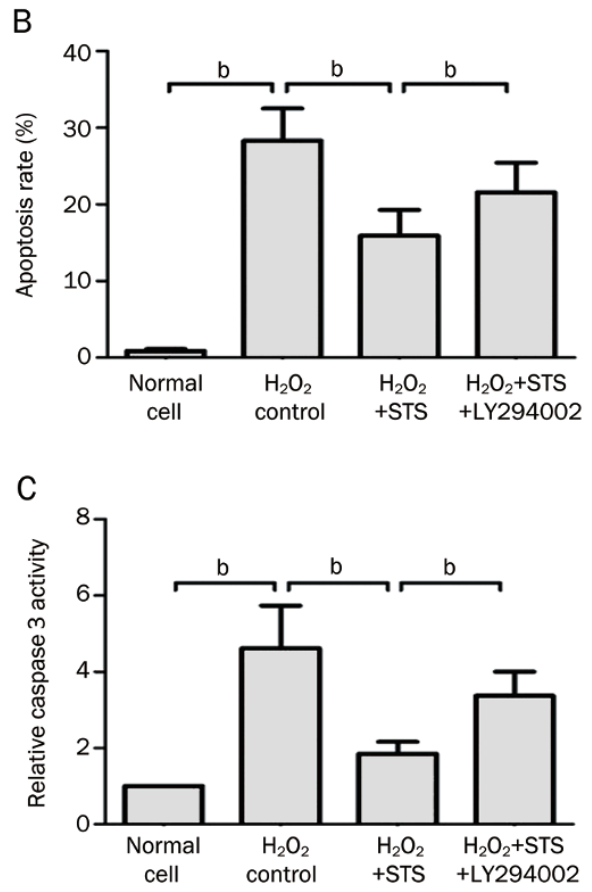

Figure 7. The $\mathrm{H}_{2} \mathrm{O}_{2}$-induced in vitro effect of STS on the apoptosis and caspase 3 activity of neonatal rat cardiac cells. (A) STS (3 $\left.\mu \mathrm{g} / \mathrm{mL}\right)$ was pretreated at $12 \mathrm{~h}$ before the incubation of cells with $200 \mu \mathrm{mol} / \mathrm{L}$ of $\mathrm{H}_{2} \mathrm{O}_{2}$ for $1 \mathrm{~h}$. Normal cells; control cells exposed to $200 \mu \mathrm{mol} / \mathrm{L}$ of $\mathrm{H}_{2} \mathrm{O}_{2}$ for $1 \mathrm{~h}$; $3 \mu \mathrm{g} / \mathrm{mL} \mathrm{STS}$ -

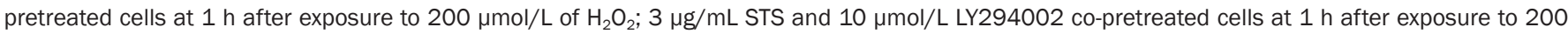
$\mu \mathrm{mol} / \mathrm{L}$ of $\mathrm{H}_{2} \mathrm{O}_{2}$. (B) Statistical analysis of the flow cytometric analysis data. (C) Statistical analysis of relative caspase 3 activity. The data represent the mean \pm SD. ${ }^{b} P<0.05$ was considered statistically significant among each group.

myocardial ischemia-reperfusion injury and attenuates $\mathrm{H}_{2} \mathrm{O}_{2}$ induced apoptosis. The cardioprotective effect of SAA is related to its ability to reduce myocardial cell apoptosis and damage induced by oxidative stress ${ }^{[25]}$. Several mechanisms have been reported to be involved in the cardioprotective effects of STS against cardiovascular injury. STS inhibits the formation of reactive oxygen species in rat heart mitochon$\mathrm{dria}^{[26]}$, inhibits $\mathrm{Ca}^{2+}$ overload in cardiomyocytes ${ }^{[24]}$, depresses $\mathrm{Na}^{+}-\mathrm{K}^{+}$-ATPase activity in cardiac-derived microsomes ${ }^{[27]}$, and improves the conditions of hemorheologic effects, including the whole blood viscosity, fibrinogen, and D-dimer ${ }^{[28]}$. A previous study has indicated the role of PI3K/Akt in mediating STS-induced cardioprotection following myocardial I/ $\mathrm{R}^{[29]}$, which is consistent with our findings. PI3K/Akt is known to mediate cytoprotective effects by regulating many downstream signals, including IKKa, eNOS, Bcl- $\mathrm{X}_{\mathrm{L}}$, p53, and GSK3. In this study, another PI3K/Akt-regulated downstream FOXO3A/Bim signal is observed in the protective effect of STS against myocardial I/R injury. Hence, there may be existing cross-talk between Akt/ FOXO3a/Bim and other Akt/ IKKa, Akt/eNOS, Akt/Bcl-X $\mathrm{L}_{\mathrm{L}}$ Akt/p53, Akt/GSK-3 signals involved in the protective effect of STS, which is indicative of a protective, multi-pathway signaling mechanism.

Activated Akt (p-Akt) can block apoptosis by inactivating several targets, including Bad, forkhead transcription factors, or caspase- ${ }^{[30]}$. In this investigation, LY294002 attenuated STS-mediated protection, indicating that PI3K/Akt pathway plays an important role in the protective effect elicited by STS. One issue that was also addressed in this study is that p-Akt is partially inhibited by LY294002 (Figure 3). Thus, this study demonstrates that STS activates Akt by inducing its phosphorylation. Then, activated p-Akt phosphorylates and inactivates FOXO3A, which alleviates the apoptosis driven by a Bim-mediated signaling pathway. However, this finding is just the tip of the iceberg. As described by Alloatti G et al ${ }^{[31]}$, the anti-apoptotic effect can also be blocked by inhibiting the PKC, PI3K, or ERK1/2 pathways. Therefore, it is possible that the protective effect elicited by STS might be mediated by multiple signaling pathways, a possibility that requires further investigation in future studies.

The FOXO subfamily of forkhead transcription factors represents a downstream target of Akt. FOXO transcription factors have been implicated in regulating diverse cellular functions, including differentiation, metabolism, proliferation, and apoptosis $^{[7,32,33]}$. The overexpression of constitutively active FOXO3a can induce BIM expression and apoptosis in the presence of survival signaling ${ }^{[8]}$. Akt phosphorylates FOXO3a at Thr 32, Ser 253, and Ser $315^{[34,35]}$, resulting in its nuclear exclusion and its association with 14-3-3 as well as the loss of target gene activation that includes the proapoptotic Bim gene ${ }^{[35-37]}$. In this study, STS treatment significantly increased the levels of p-Akt (activation) and induced the phosphorylation (inactivation) of FOXO3A in I/R rat cardiomyocytes and $\mathrm{H}_{2} \mathrm{O}_{2-}$ treated neonatal rat cardiac cells. This FoxO3A inactivation 
was accompanied by significant decreases in the expression of one key FOXO3A target gene, the proapoptotic Bim. Thus, the possible mechanism underlying the protective effect of STS against rat myocardial ischemia-reperfusion apoptosis injury might be elicited via the activation of a PI3K/Akt/FOXO3A/ Bim-mediated signaling pathway as follows: (1) The STS activates the PI3K/Akt pathway via the phosphorylation of Akt; (2) p-Akt inactivates FOXO3A through the phosphorylation of FOXO3A; (3) The phosphorylation of FOXO3A can induce its nuclear-to-cytoplasmic translocation, thereby inhibiting its transcription factor activity; and (4) The displacement of FOXO3A from the nucleus into the cytoplasm inhibits its transcriptional activity, and in turn, the expression of its downstream target molecule Bim, which is responsible for the apoptosis-related activation of caspase- 3 and cytochrome $c$ release. However, whether the activation of the PI3K/Akt/FOXO3A/ Bim pathway is the only way to inhibit cardiomyocyte apoptosis during I/R remains unknown. The full characterization of other signaling pathways needs to be further investigated.

Another issue that has not been investigated in this study is whether the activities of STS require an intracellular receptor. The PI3K/Akt pathway plays an important role in I/R injury, and the possible involvement of this pathway in the cardioprotective effect of STS warrants further investigation. It is conceivable that there is a tyrosine kinase in the putative STS receptor that activates the PI3K/Akt pathway. The elucidation of this possibility will require an intensive exploration of the STS receptor.

The salient finding of this study is that STS pretreatment elicits a significant cardioprotective effect in reducing infarct size, improving cardiac function, and inhibiting apoptosis - either directly or indirectly - through the PI3K/Akt/FOXO3A/ Bim pathway. These data suggest that the application of STS represents a new therapeutic modality for treating myocardial $\mathrm{I} / \mathrm{R}$ injury. A complete understanding of the mechanisms underlying the protective effects of STS will greatly facilitate the realization of its clinical potential.

\section{Acknowledgements}

This study was supported by the 2012 Zhejiang Province Traditional Chinese Medicine Scientific Research Fund (A) (2012ZA015).

\section{Author contribution}

Chang-lin ZHAI designed research; Mei-qi ZHANG, and Yueliang ZHENG performed research; Huan CHEN, Jian-feng TU, Jing-jie CHAI, Ye SHEN, and Jun-ping GUO contributed new analytical tools and reagents; Xiang-hong YANG, Shuren YUAN, and Liang-zhong CHEN analyzed data; Mei-qi ZHANG and Jian-hong LU wrote the paper.

\section{Abbreviations}

Sodium tanshinone IIA sulfonate, STS; ischemia-reperfusion, I/R; infarction size, IS; area at risk, AAR; terminal deoxynucleotidyl transferase-mediated dUTP-biotin nick end labeling, TUNEL; phosphatidylinositol-3-OH kinase, PI3K; forkhead box $\mathrm{O}, \mathrm{FOXO}$; heart rate, $\mathrm{HR}$, the first derivative of developed pressure, $\mathrm{d} p / \mathrm{d} t$; left ventricular developed pressure, LVDP; coronary flow, CF; left ventricles, LVS; phosphatidylinositol4,5-bisphosphate, PIP2; 3-phosphoinositide-dependent protein kinase-1, PDK1.

\section{References}

1 Choi H, Kim SH, Chun YS, Cho YS, Park JW, Kim MS. In vivo hyperoxic preconditioning prevents myocardial infarction by expressing bcl-2. Exp Biol Med 2006; 231: 463-72.

2 Tian Y, Zhang W, Xia D, Modi P, Liang D, Wei M. Postconditioning inhibits myocardal apoptosis during prolonged reperfusion via a JAK2STAT3-Bcl-2 pathway. J Biomed Sci 2011; 18: 53.

3 Zhai CL, Zhang MQ, Zhang Y, Xu HX, Wang JM, An GP, et al. Glycyrrhizin protects rat heart against ischemia-reperfusion injury through the blockade of HMGB1-dependent phospho-JNK/Bax pathway. Acta Pharmacol Sin 2012; 33: 1477-87.

4 Freude B, Masters TN, Robicsek F, Fokin A, Kostin S, Zimmermann $\mathrm{R}$, et al. Apoptosis is initiated by myocardial ischemia and executed during reperfusion. J Mol Cell Cardiol 2000; 32: 197-208.

5 Hausenloy DJ, Yellon DM. New directions for protecting the heart against ischaemia-reperfusion injury: targeting the reperfusion injury salvage kinase (RISK)-pathway. Cardiovasc Res 2004; 61: 448-60.

6 Skurk C, Izumiya Y, Maatz H, Razeqhi P, Shiojima I, Sandri M, et al. The FOXO3a transcription factor regulates cardiac myocyte size downstream of AKT signaling. J Biol Chem 2005; 280: 20814-23.

7 Yang JY, Xia W, Hu MC. Ionizing radiation activates expression of FOXO3a, Fas ligand, and Bim, and induces cell apoptosis. Int J Oncol 2006; 29: 643-8.

8 Yang JY, Hung MC. Deciphering the role of the forkhead transcription factors in cancer therapy. Curr Drug Targets 2011; 12: 1284-90.

9 Weston CR, Balmanno K, Chalmers C, Hadfield K, Molton SA, Lev R, et al. Activation of ERK1/2 deltaRaf-1: ER* represses Bim expression independently of the JNK or PI3K pathways. Oncogene 2003; 22: 1281-93.

10 Zhou GY, Zhao BL, Hou JW, Ma GE, Xin WJ. Protective effects of sodium tanshione IIA sulphonate against adriamycin-induced lipid peroxidation in mice hearts in vivo and in vitro. Pharmacol Res 1999; 40: 487-91.

11 Shang Q, Xu H, Huang L. Tanshinone IIA: a promising natural cardioprotective agent. Evid Based Complement Alternat Med 2012. Doi: 10.1155/2012/716459.

12 Cheng TO. Cardiovascular effects of Danshen. Int J Cardiol 2007; 121: 9-22.

13 Jiang B, Zhang L, Wang Y, Li M, Wu W, Guan S, et al. Tanshinone IIA sodium sulfonate protects against cardiotoxicity induced by doxorubicin in vitro and in vivo. Food Chem Toxicol 2009; 47: 153844.

14 Yang R, Liu A, Ma X, Li L, Su D, Liu J. Sodium tanshinone IIA sulfonate protects cardiomyocytes against oxidative stress-mediated apoptosis through inhibiting JNK activation. J Cardiovasc Pharmacol 2008; 51 : 396-401.

15 Juhasz B, Thirunavukkarasu M, Pant R, Zhan L, Penumathsa SV, Secor $\mathrm{ER} \mathrm{Jr}$, et al. Bromelain induces cardioprotection against ischemiareperfusion injury through Akt/FOXO pathway in rat myocardium. Am J Physiol Heart Circ Physiol 2008; 294: H1365-70.

16 Penumathsa SV, Koneru S, Thirunavukkarasu M, Zhan L, Prasad K, Maulik N. Secoisolariciresinol diglucoside: relevance to angiogenesis and cardioprotection against ishcemia-reperfusion injury. J Pharmacol Exp Ther 2007; 320: 951-9. 
17 Penumathsa SV, Thirunavukkarasu M, Koneru S, Juhasz B, Zhan L, Pant $\mathrm{R}$, et al. Statin and resveratrol in combination induces cardioprotection against myocardial infarction in hypercholesterolemic rat. J Mol Cell Cardiol 2007; 42: 508-16.

$18 \mathrm{Hu} \mathrm{X,} \mathrm{Zhou} \mathrm{X,} \mathrm{He} \mathrm{B,} \mathrm{Xu} \mathrm{C,} \mathrm{Wu} \mathrm{L,} \mathrm{Cui} \mathrm{B,} \mathrm{et} \mathrm{al.} \mathrm{Minocycline} \mathrm{protects}$ against myocardial ischemia and reperfusion injury by inhibiting high mobility group box 1 protein in rats. Eur J Pharmacol 2010; 638: 84-9.

19 Sgobbo P, Pacelli C, Grattagliano I, Villani G, Cocco T. Carvedilol inhibits mitochondrial complex I and induces resistance to $\mathrm{H} 2 \mathrm{O} 2$ mediated oxidative insult in $\mathrm{H} 9 \mathrm{C} 2$ myocardial cells. Biochim Biophys Acta 2007; 1767: 222-32.

$20 \mathrm{Kim} \mathrm{CH}$, Hao J, Ahn HY, Kim SW. Activation of Akt/protein kinase $B$ mediates the protective effects of mechanical streching against myocardial ischemia-reperfusion injury. J Vet Sci 2012; 13: 235-44.

21 Hong HJ, Liu JC, Chen JJ, Chan P, Cheng TH. Tanshinone IIA prevents doxorubicin-induced cardiomyocyte apoptosis through Akt-dependent pathway. Int J Cardiol 2012; 157: 174-9.

22 Zhang Y, Wei L, Sun D, Cao F, Gao H, Zhao L, et al. Tanshinone IIA pretreatment protects myocardium against ischaemia/reperfusion injury through the phosphatidylinositol 3-kinase/Akt-dependent pathway in diabetic rats. Diabetes Obes Metab 2010; 12: 316-22.

$23 \mathrm{Li} \mathrm{XH}$, Tang RY. Relationship between inhibitory action of tanshinone on neutrophil function and its prophylactic effects on myocardial infarction. Zhongguo Yao Li Xue Bao 1999 ; 12: 269-72.

24 Shanghai Cooperative Group for the Study of Tanshinone IIA. Therapeutic effect of sodium tanshinone IIA sulfonate in patients with coronary heart diseases: a double blind study. J Tradit Clin Med 1984; 4: 20-4.

25 Fan HY, Yang L, Fu FH, Xu H, Meng QG, Zhu HB, et al. Cardioprotective effects of salvianolic acid a on myocardial ischemia-reperfusion injury in vivo and in vitro. Evidence-based complementary and alternative medicine. 2011; doi: 10.1155/2012/508938.

26 Zhou G, Jiang W, Zhao Y, Ma G, Xin W, Yin J, et al. Sodium tanshinone IIA sulfonate mediates electron transfer reaction in rat heart mitochondria. Biochem Pharmacol 2003; 65: 51-7.
27 Xu CQ, Cang JS, He XM. Inhibition effect of tanshinone IIA on electron current of L-calcium in rat heart cell. Acta Pharmacol Toxicol Sin 1996; 12: 85-8.

28 Wang YL, Chen NH, Ding JH. Inhibitory effects of sodium tanshinone IIA sulfonate on microsomal $\mathrm{Na}^{+}, \mathrm{K}^{+}$-ATPase in rat heart and brain. Acta Pharmacol Toxicol Sin 1994; 8: 19-23.

29 Zuo YJ, Li YQ, Wang G. Effect of tanshinone IIA sulfonic acid natrium injection on angina pectoris and hemorrheology in patients with coronary artery disease. Prog Mod Biomed 2007; 7: 732-4.

30 Zhao H, Sapolsky RM, Steinberg GK. Phosphoinositide-3-kinase/Akt survival pathways are implicated in neuronal survival after stroke. Mol Neurobiol 2006; 34: 249-70.

31 Alloatti G, Arnoletti E, Bassino E, Penna C, Perrelli MG, Ghe C, et al. Obestatin affords cardioprotection to the ischemic-reperfused isolated rat heart and inhibits apoptosis in cultures of similary stressed cardiomyocytes. Am J Physiol Heart Circ Physiol 2010; 299: H47081.

32 Accili D, Arden KC. FoxOs at the crossroads of cellular metabolism, differentiation, and transformation. Cell 2004; 117: 421-6.

33 Skurk C, Maatz H, Kim HS, Yang J, Abid MR, Aird WC, et al. The Aktregulated forkhead transcription factor FOXO3a controls endothelial cell viability through modulation of the caspase-8 inhibitor FLIP. J Biol Chem 2004; 279: 1513-25.

34 Greer EL, Brunet A. FOXO transcription factors at the interface between longevity and tumor suppression. Oncogene 2005; 24: 7410-25.

35 Brunet A, Bonni A, Ziqmond MJ, Lin MZ, Juo P, Hu LS, et al. Akt promotes cell survival by phosphorylating and inhibiting a Forkhead transcriptin factor. Cell 1999; 96: 857-68.

36 Gilley J, Coffer PJ, Ham J. FOXO transcription factors directly activate bim gene expression and promote apoptosis in sympathetic neurons. J Cell Biol 2003; 162: 613-22.

37 Urbich C, Knau A, Fichtlscherer S, Walter DH, Bruhl T, Potente M, et al. FOXO-dependent expression of the proapoptotic protein Bim: Pivotal role for apoptosis signaling in endothelial progenitor cells. FASEB $J$ 2005; 19: 974-6. 